\title{
Functional biopolymer-based matrices for modulation of chronic wound enzyme activities
}

\author{
Antonio Francesko ${ }^{a}$, Diana Soares da Costa ${ }^{\text {b,c }}$, Rui L. Reis ${ }^{\text {b,c }}$, Iva Pashkuleva ${ }^{\text {b,c }}$, Tzanko Tzanov ${ }^{a, *}$ \\ ${ }^{a}$ Group of Molecular and Industrial Biotechnology, Department of Chemical Engineering, Universitat Politècnica de Catalunya, Rambla Sant Nebridi s/n, 08222 Terrassa, Spain \\ b 3B's Research Group, University of Minho, Headquarters of the European Institute of Excellence on Tissue Engineering and Regenerative Medicine, AvePark, 4806-909 Taipas, \\ Guimarães, Portugal \\ 'ICVS/3B's - PT Government Associate Laboratory, Braga/Guimarães, Portugal
}

\section{A R T I C L E I N F O}

\section{Article history:}

Received 5 June 2012

Received in revised form 2 October 2012

Accepted 7 October 2012

Available online 13 October 2012

\section{Keywords:}

Collagen

Hyaluronic acid

Chitosan

Polyphenols

Chronic wounds

\begin{abstract}
A B S T R A C T
Collagen, collagen/hyaluronic acid (HA) and collagen/HA/chitosan (CS) sponges loaded with epigallocatechin gallate (EGCG), catechin (CAT) and gallic acid (GA) were developed and evaluated as active chronic wound dressings. Their physico-mechanical properties, biostability, biocompatibility and ability to inhibit in vitro myeloperoxidase (MPO) and collagenase-major enzymes related with the persistent inflammation in chronic wounds-were investigated as a function of the biopolymer composition and the polyphenolic compound used. The results demonstrated that the molecular weight of HA influences significantly the bulk properties of the obtained materials: higher elastic modulus, swelling ability and biostability against collagenase were measured when HA with higher molecular weights ( 830 and $2000 \mathrm{kDa})$ were added to the collagen matrices. The addition of CS and the polyphenols increased further the biostability of the sponges. Preliminary in vitro tests with fibroblasts revealed that the cells were able to adhere to all sponges. Cell viability was not affected significantly by the addition of the polyphenols; however, the presence of CS or high molecular weight HA in the sponge composition was associated with lower cellular viability. Finally, all specimens containing polyphenols efficiently inhibited the MPO activity. The highest inhibition capacity was observed for EGCG $\left(I_{50}=15 \pm 1 \mu \mathrm{M}\right)$ and it was coupled to the highest extent of binding to the biopolymers (>80\%) and optimal release profile from the sponges that allowed for prolonged (up to 3-5 days) effects.
\end{abstract}

(C) 2012 Acta Materialia Inc. Published by Elsevier Ltd. All rights reserved.

\section{Introduction}

Chronic (non-healing) wounds are characterized by a persistent inflammation during which numerous mutually causative cellular events lead to overexpression of proteolytic and neutrophil-derived oxidative enzymes. For example, total matrix metalloproteinases (MMPs) activity, being predominantly collagenases, is up to 30 -fold greater in chronic than in acute wound fluids [1], resulting in a protease/antiprotease imbalance and excessive breakdown of the extracellular matrix (ECM). The proteolytic damage of the tissue is further promoted by oxidation of the natural protease inhibitors with hypochlorous acid $(\mathrm{HOCl})$ generated by the main neutrophil enzyme, myeloperoxidase (MPO) [2]. It is believed that bringing the counts of the deleterious chronic wound enzymes down to the levels found in acute wounds would allow healing to progress [3]. Several approaches have been proposed and different biopolymer-based dressings have been developed in order to achieve this effect. Among them, collagen-the most abundant

\footnotetext{
* Corresponding author. Tel.: +34 937398570; fax: +34 937398225.

E-mail address: tzanko.tzanov@upc.edu (T. Tzanov).
}

structural component of the ECM-is largely employed in dressings to create optimal moist conditions for wound healing and absorb proteases from wound exudates [4]. In addition, collagen ensures fibroblasts adhesion and growth, leading to the formation of new granulation tissue and epithelium at the wound site [5]. Hyaluronic acid (HA), a non-sulfated glycosaminoglycan (GAG), is another important moisturizing constituent of the ECM. HA macromolecules inhibit the expression of some MMPs to a different extent, depending on their molecular weight [6]. Chitosan (CS), sharing structural similarities with naturally occurring GAGs, possesses intrinsic antimicrobial properties and accelerates early phase wound healing [7]. The assembling of collagen, HA and/or CS into composite materials has displayed advantages in tissue regeneration applications over the use of any of these biopolymers alone $[8,9]$.

Wound dressings, on the other hand, require high stability during the extended treatment duration combined with low frequency of dressing changes as recommendable for chronic wounds treatment. This stability-at-use might be compromised by the intrinsic susceptibility of the biopolymer components to enzymatic degradation in contact with biological fluids. The application of suitable 
chemical cross-linkers, e.g. N-(3-dimethylaminopropyl)-N'ethylcarbodiimide hydrochloride (EDAC), usually applied in combination with N-hydroxysuccinimide (NHS) to form "zero length" bonds between polymers [10], overcomes their enzymatic degradability. Moreover, the multifactorial nature of the chronic wounds does also require upgrading of the biopolymer platforms with active agents to more efficiently interfere at molecular level with the biochemical events governing the ECM breakdown. The latter can be achieved by topical application of bioactive molecules, e.g. enzyme inhibitors.

Natural polyphenolic compounds recently received a great deal of attention in medicine owing to their antioxidant, antimicrobial, anti-inflammatory and consequently wound healing promoting properties [11]. Their ability to complex with proteins and polysaccharides has been exploited for improving the biopolymers stability to enzymatic degradation [12]. Moreover, the propensity of these compounds to bind proteins presumably accounts for the fact that polyphenols were able to inhibit virtually every enzyme tested in vitro [11]. Our previous studies indicated that controlled inhibition of major chronic wound enzymes could be achieved by the application of plant polyphenolic extracts $[13,14]$.

The current study focuses on the development of a sponge-like material that combines the intrinsic properties of the individual biopolymers-collagen, hyaluronic acid and chitosan-in a composite dressing and the optimization of the biopolymer composition of such dressing for chronic wound treatment. The obtained sponges were additionally loaded with bioactive polyphenols from Camellia sinensis to modulate the activity of chronic wound enzymes and further enhance the biostability of the materials. Tuning of the physico-mechanical, biological and functional properties of the composite sponges is discussed as a function of the biopolymer composition and the used bioactive agent.

\section{Materials and methods}

\subsection{Materials}

Collagen type I from bovine skin was kindly supplied by Lohmann \& Rauscher International GmbH \& Co. (Germany). Hyaluronic acid sodium salts of different molecular weights (Mw; 6830 and $2000 \mathrm{kDa}$ ) from Lifecore Biomedical (USA) and ultrapure chitosan (Mw $\sim 50 \mathrm{kDa}$ ) from KitoZyme (Belgium) were used for the experiments. Collagenase from Clostridium histolyticum (277.50 $\mathrm{U} \mathrm{mg}^{-1}$ solid, one unit liberates peptides from collagen equivalent in ninhydrin colour (at $570 \mathrm{~nm}$ ) to $1.0 \mu \mathrm{mol}$ of leucine in $5 \mathrm{~h}$ at $\mathrm{pH} 7.4$ and $37{ }^{\circ} \mathrm{C}$ in the presence of calcium ions), $\mathrm{N}$-(3-[2-Furyl]Acryloyl)Leu-Gly-Pro-Ala (FALGPA), green tea polyphenols (epigallocatechin gallate, catechin and gallic acid), $\mathrm{N}$-(3-dimethylaminopropyl)$\mathrm{N}^{\prime}$-ethylcarbodiimide hydrochloride (EDAC) and $\mathrm{N}$ hydroxysuccinimide (NHS) were purchased from Sigma-Aldrich. Highly purified MPO from human leukocytes $\left(1550 \mathrm{U} \mathrm{mg}^{-1}\right.$ solid, one unit will produce an increase in absorbance at $470 \mathrm{~nm}$ of $1.0 \mathrm{~min}^{-1}$ at $\mathrm{pH} 7.0$ and $25^{\circ} \mathrm{C}$, calculated from the initial rate of reaction using guaiacol substrate) was obtained from Planta Natural Products (Austria). EnzChek ${ }^{\circledR}$ kit was purchased from Life Technologies (Spain). All other reagents were of analytical grade purchased from Sigma-Aldrich and used as received.

\subsection{Preparation of biopolymer sponges}

All individual biopolymer solutions were prepared at a concentration of $1 \%(\mathrm{w} / \mathrm{v})$. Collagen and CS were dissolved in $1 \%$ acetic acid, while HAs were dissolved in distilled water. The prepared solutions were mixed as follows: collagen/hyaluronic acid (CHA) in 9:1 weight ratio, and collagen/hyaluronic acid/chitosan (CHACS) in the ratio $4.5: 1: 4.5$. The mixtures were homogenized for $20 \mathrm{~min}$, poured in Petri dishes with $3 \mathrm{~mm}$ thickness and freeze-dried. The obtained sponges were cross-linked using $96 \%$ ethanol containing EDAC $(50 \mathrm{mM}) / \mathrm{NHS}(10 \mathrm{mM})$ for $24 \mathrm{~h}$, thoroughly washed with distilled water and freeze-dried again. CHA specimens were named after the Mw of the used HA (CHA6, CHA830, CHA2000). The CHACS specimen was prepared using HA with Mw of $830 \mathrm{kDa}$.

\subsection{Loading of the biopolymer sponges with polyphenols}

The cross-linked sponges $(10 \mathrm{mg})$ were loaded with polyphenols ( $1 \mathrm{ml}$ of $1 \mathrm{mM}$ aqueous solutions of epigallocatechin gallate (EGCG), catechin (CAT) or gallic acid (GA)) by simple immersion at $4{ }^{\circ} \mathrm{C}$ for $24 \mathrm{~h}$. The impregnation time was chosen according to the literature to ensure equilibrium loading of the polyphenols [15]. Thereafter the samples were freeze-dried.

\subsection{Characterization of the cross-linked biopolymer sponges}

\subsubsection{Fourier transform infrared spectroscopy (FTIR)}

Infrared spectra of the sponges were recorded using a Perkin Elmer Spectrum 100 FT-IR spectrometer utilizing the attenuated total reflectance technique. Spectra were averaged over 75 scans.

\subsubsection{Tensile testing}

Strip-shaped sponges $(30 \times 10 \times 3 \mathrm{~mm})$ were subjected to uniaxial tensile testing in a wet state at room temperature using a universal mechanical testing machine INSTRON 5540. After removing the excess of phosphate buffer saline (PBS, pH 7.4) by gentle contact with filter paper, the specimens were stretched at a cross-head speed of $5 \mathrm{~mm} \mathrm{~min}^{-1}$ until failure. Five samples were tested per each experimental group.

\subsection{Extent of collagenase adsorption and biostability of the biopolymer matrices}

Collagenase binding onto the cross-linked sponges was studied in $50 \mathrm{mM}$ PBS ( $\mathrm{pH} 7.4,40 \mathrm{mM} \mathrm{CaCl}_{2}$ ) containing $2 \mu \mathrm{g} \mathrm{ml}^{-1}$ collagenase incubated for $24 \mathrm{~h}$ at $37^{\circ} \mathrm{C}$. Thereafter, the supernatants were collected and analysed for the remaining collagenase activity using EnzChek $^{\circledR}$. Briefly, the remaining in the supernatants collagenase was left to cleave a gelatin-fluorescein conjugate in darkness at room temperature for $6 \mathrm{~h}$, after which the fluorescence was measured (495/515 nm) using a microplate reader Infinite M200, Tecan (Austria). The results are expressed as the percentage of collagenase binding onto the materials $(n=5)$, inversely proportional to the enzymatic activity in the supernatant.

Biodegradation tests were carried out in vitro by incubation of

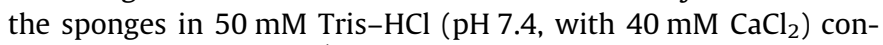
taining 10 or $60 \mu \mathrm{g} \mathrm{ml}^{-1}$ collagenase at $37{ }^{\circ} \mathrm{C}$ for $24 \mathrm{~h}$. After complete hydrolysis of the supernatants in $6 \mathrm{~N} \mathrm{HCl}$ for $24 \mathrm{~h}$ at $90^{\circ} \mathrm{C}$ and neutralization with $6 \mathrm{~N} \mathrm{NaOH}$, the hydroxyproline (Hyp) content was determined using the method previously described by Reddy and Enwemeka [16]. The calculation was based on the assumption that collagen contains $\sim 14 \%$ Hyp [17]. Five samples were tested per each experimental group.

\subsection{Polyphenols binding onto the biopolymer sponges and release studies}

Polyphenols uptake by the sponges was estimated by comparison of the total phenol content in solution before and after incubation with sponges $(n=3)$ using the Folin-Ciocalteu spectrophotometric method [18]. The calibration curves were prepared using different concentrations of polyphenols. The experiments for the cumulative release of polyphenols were started by 
immersing $5 \mathrm{mg}$ of specimens in $500 \mu \mathrm{d}_{2} \mathrm{O}$. At defined time periods the sponges were transferred into the same amount of fresh $\mathrm{dH}_{2} \mathrm{O}$. The total phenol content for three samples per each experimental group was determined as described above.

\subsection{Swelling behaviour of the biopolymer dressings}

Specimens with and without polyphenols were evaluated for their ability to absorb physiological fluid [19]. The sponges were first weighed $\left(W_{\mathrm{i}}\right)$ and then immersed in PBS $\mathrm{pH} 7.4$ for $24 \mathrm{~h}$. The swollen samples were withdrawn and weighed again without dripping $\left(W_{s}\right)$. Their capacity to absorb physiological fluid was defined as the ratio between the retained fluid $\left(W_{s}-W_{i}\right)$ and the dry weight of the sample $\left(W_{i}\right)$. Five samples of each specimen were evaluated for swelling.

\subsection{Enzymes inhibition assays}

Inhibition of collagenase activity by the polyphenols used in the study was determined using FALGPA as a substrate [20]. Collagenase $\left(5 \mu \mathrm{g} \mathrm{ml}^{-1}\right)$ hydrolysis of FALGPA $(1 \mathrm{mM})$ incubated with different concentrations of polyphenols for $1 \mathrm{~h}$ in $50 \mathrm{mM}$ Tricine (pH 7.5, $100 \mathrm{mM} \mathrm{CaCl}_{2}$ and $400 \mathrm{mM} \mathrm{NaCl}$ ) was monitored at $345 \mathrm{~nm}$ during $5 \mathrm{~min}$.

The inhibitory effect of the polyphenols on MPO activity was measured using guaiacol as a substrate. Different polyphenol concentrations were incubated for $1 \mathrm{~h}$ with MPO $(0.60 \mathrm{U})$ and guaiacol $(167 \mathrm{mM})$ buffered with $500 \mu \mathrm{l}$ of $50 \mathrm{mM}$ PBS pH 6.6 at $37^{\circ} \mathrm{C}$. Thereafter, $270 \mu \mathrm{l}$ aliquots were placed in a 96-well microplate and the enzymatic reaction was started by adding $30 \mu \mathrm{l}$ of $10 \mathrm{mM} \mathrm{H}_{2} \mathrm{O}_{2}$. The change in absorbance at $470 \mathrm{~nm}$ was monitored and the activity determined by the rate of absorbance increase per min. The capacity of the polyphenols to inhibit enzyme activities are expressed as the concentrations required for the inhibition of $50 \%$ enzymatic activities (IC50, $n=3$ ).

The inhibitory effect of the polyphenols released from the biopolymer sponges on MPO activity was determined after specimens $(2 \mathrm{mg})$ incubation with the enzyme $(0.60 \mathrm{U})$. After different incubation times the enzymatic activity was determined as described above and expressed as a percentage of enzyme inhibition compared to the control (sponges without polyphenols). All measurements were carried out in triplicate.

\subsection{Cell viability, morphology and distribution}

The sponges were sterilized by ethylene oxide and hydrated in PBS for $1 \mathrm{~h}$ before seeding with L929 human fibroblast-like cell line (European Collection of Cell Cultures, UK) at 100,000 cells/sponge concentration in Dulbecco's modified Eagle's medium (DMEM; Sigma-Aldrich, USA) supplemented with $10 \%$ of fetal bovine serum (FBS; Biochrom AG, Germany) and $1 \%$ antibiotic/antimycotic (Gibco, USA). Cells were cultured for 1,2 and 3 days at $37^{\circ} \mathrm{C}$ under a humidified atmosphere of $5 \% \mathrm{CO}_{2}$.

Cell viability for each culturing time was determined by CellTiter 96 Aqueous One Solution Cell Proliferation Assay (Promega, USA). The absorbance was measured at $490 \mathrm{~nm}$ using a microplate reader (Synergie HT, Bio-Tek, USA). Three samples of each specimen were characterized per time point.

The morphology of the cells cultured in contact with the sponges was observed by scanning electron microscopy (SEM). The samples were washed twice with PBS, fixed with $2.5 \%$ glutaraldehyde for $1 \mathrm{~h}$ at $4{ }^{\circ} \mathrm{C}$ and washed again with PBS prior to dehydration in a graded series of ethanol. Finally, the sponges were dried using hexamethyldisilazane, coated with gold (sputter coater model SC502, Fisons Instruments, UK) and analysed by SEM (model
S360, Leica, Cambridge, UK) at $15 \mathrm{kV}$. Longitudinal sections were observed to evaluate distribution of cells throughout the sponge.

\subsection{Statistical analysis}

The results are presented as mean values \pm standard deviation (SD) when they followed a normal distribution. In this case, statistical analysis (Graph Pad Prism Software 5.04 for Windows, USA) by a one-way analysis of variance (ANOVA) followed by a post hoc Tukey test was performed to test the differences among the samples. The data are presented as box plots when they did not follow a normal distribution (Shapiro-Wilk test). The Mann-Whitney test was applied in this case in order to determine the statistical significance of the observed differences. Throughout the discussion, the differences were considered significant if $P<0.01$.

\section{Results and discussion}

\subsection{Characterization of the biopolymer matrices}

\subsubsection{FTIR analysis}

EDAC-mediated cross-linking can undergo two distinct pathways depending on the available functional groups in polymers: either formation of amide linkages by activation of carboxylic moieties to react with free amines, or an acid anhydride formation from two carboxyl groups and further reaction with hydroxyl groups to yield ester bonds, may occur [21]. Both pathways are likely to be followed for CHAs and CHACS to form inter- and intra-molecular cross-links between the biopolymers (e.g. some examples of intermolecular cross-linking are shown in Scheme 1).

After collagen treatment with EDAC, an increase in the intensity of the amide $\mathrm{I}\left(\mathrm{C}=\mathrm{O}\right.$ stretching at $\left.1630 \mathrm{~cm}^{-1}\right)$ and amide II $(\mathrm{N}-\mathrm{H}$ bending at $1543 \mathrm{~cm}^{-1}$ ) bands was observed (Fig. $1 \mathrm{~A}, \mathrm{~b}$ ). These signals together with the less intensive symmetric stretching band of carboxylate salts at $1399 \mathrm{~cm}^{-1}$ and the ester band with higher intensity at $1079 \mathrm{~cm}^{-1}$ confirmed the covalent cross-linking of the biopolymer. The intensity of the ester band became more pronounced in the spectra of the cross-linked CHA sponges, for simplicity represented only with CHA830 spectrum (Fig. 1A, c). This indicated a higher esterification yield for these samples compared to collagen alone. In addition, the changes were more pronounced in CHA sponges containing higher Mw HA compared to CHA6 (Fig. 1B). The latter observation could be explained with the higher number of carboxylic groups in a single molecule that increases the probability of acid anhydride formation mediated by EDAC and thus subsequent ester bonding. On the other hand, the increased involvement of the carboxyl groups in these specimens to form acid anhydrides led to the lower extent of amide cross-linking, confirmed by the lower intensities of the amide I and amide II bands compared to CHA6. Finally, with the addition of chitosan to CHA830 sponge, a pronounced peak attributed to the mixed stretching and bending vibrations of the $\mathrm{C}-\mathrm{O}-\mathrm{C}$ bond (1150$970 \mathrm{~cm}^{-1}$ ) appeared and overlapped the ester peak (Fig. 1A, d). The FTIR spectrum of CHACS hybrid sponge is rather complicated and shows overlapping effects of all three biopolymers after cross-linking.

\subsubsection{Mechanical properties of the sponges}

The results from uniaxial tensile tests performed on the cross-linked biopolymer sponges showed an increase in both the material elastic modulus and failure strain for CHA and CHACS specimens compared to the pure collagen sponge (Fig. 2). Although amongst CHAs the modulus did not differ significantly, its values were higher for CHA830 and CHA2000 compared to CHA6 (Fig. 2A). The elasticity of the composite matrices further increased 

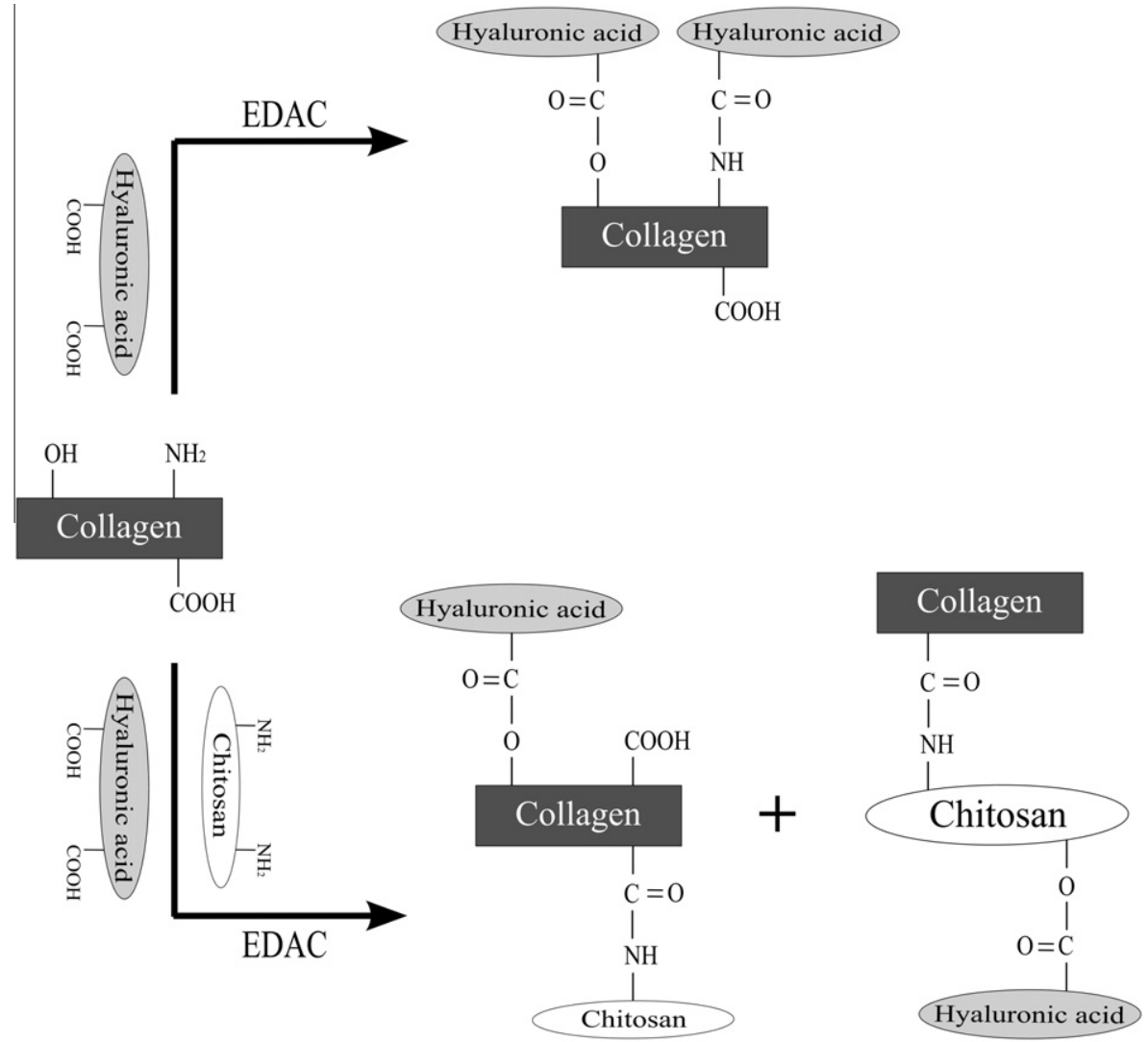

Scheme 1. Cross-linking of the biopolymers with EDAC.
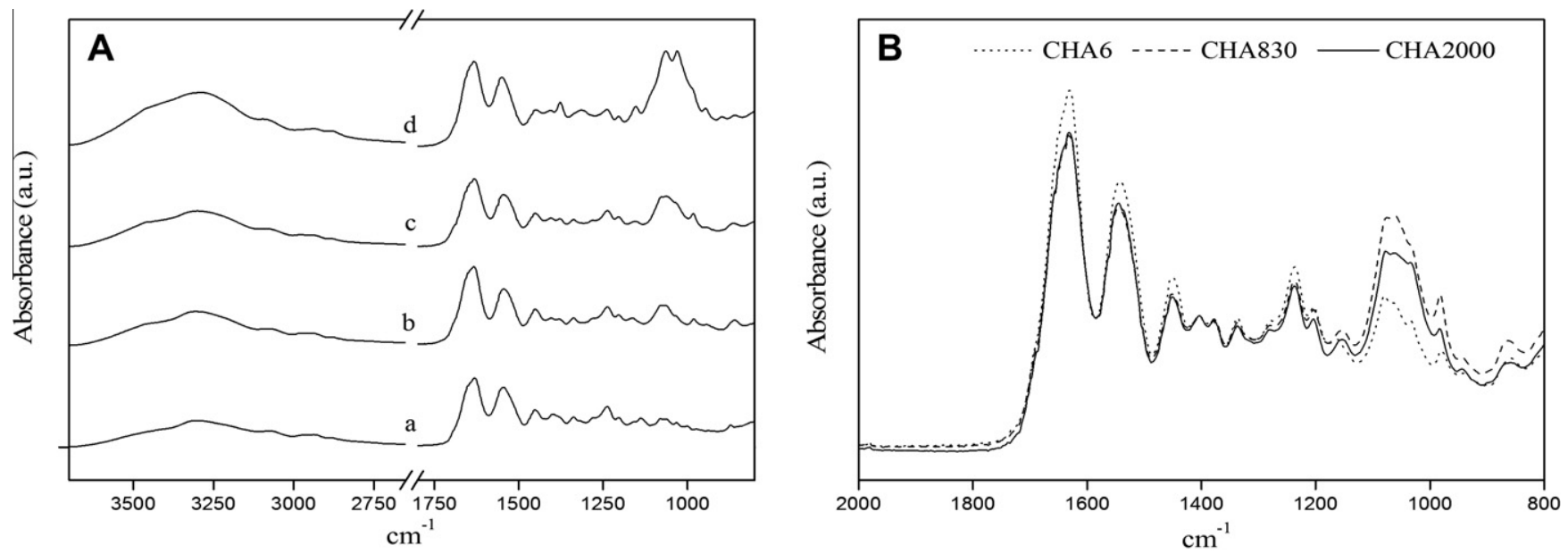

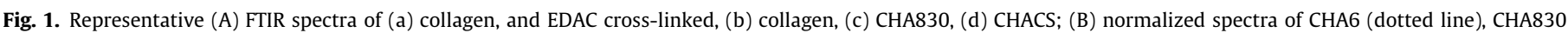
(dashed line), CHA2000 (solid line).

with the addition of chitosan. Similarly, CHA specimens with higher Mw and CHACS displayed significant $(P<0.001)$ increase in the strain at break, while those of CHA6 were not considerably different from the cross-linked collagen (Fig. 2B).

Unlike collagen in biological tissues, the materials prepared from collagen extracts normally possess low tensile strength. Cross-linking is widely used to improve the elastic modulus of polymer materials, although this negatively affects their stretchability. To overcome this problem for biomaterials in close contact with body tissues, hydrophilic in nature polymers could be used in combination with cross-linking, resulting in increased water retention capacity and energy storage of the matrices, and consequently, higher stretchability and tensile strength [22]. This study confirms that the addition of polysaccharides, such as chitosan and high molecular weight hyaluronic acid, into collagen-based matrices, in combination with chemical cross-linking, is an efficient strategy to improve both elastic modulus and material stretchability.

\subsection{Extent of collagenase adsorption and biostability of the biopolymer sponges}

In acute wounds the collagenases contribute to the controlled reconstruction of the damaged skin during wound closure and remodelling. In chronic wounds, however, their elevated levels 

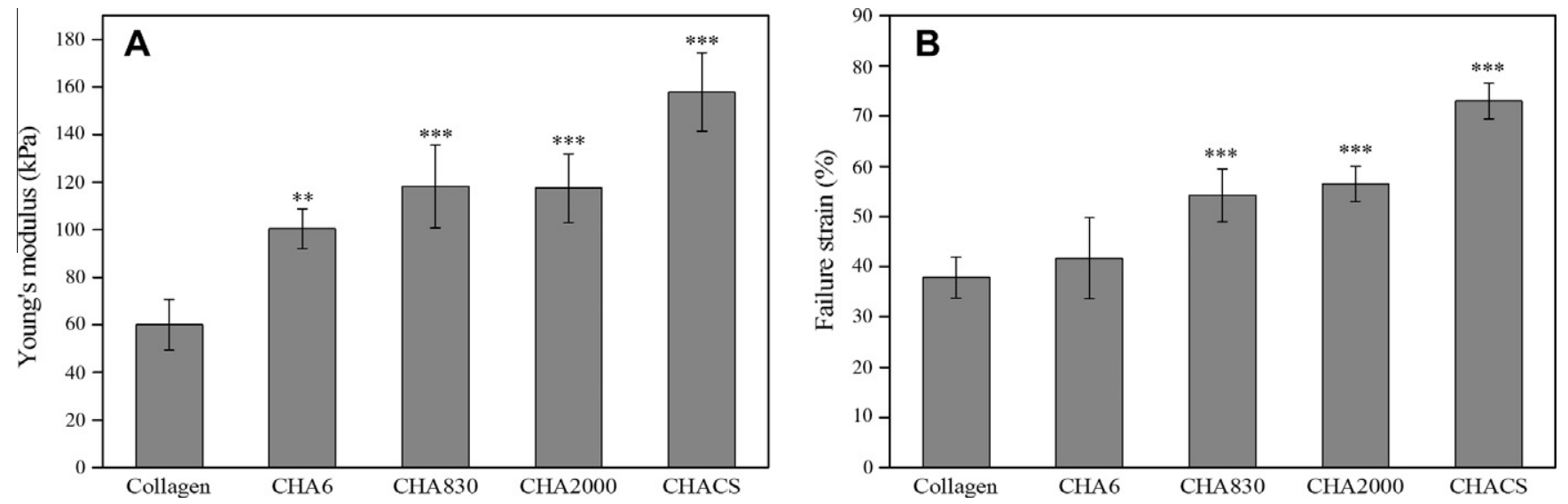

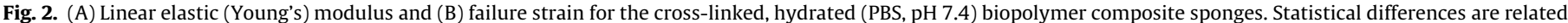
to collagen and represented as ${ }^{* *} P<0.01$ and ${ }^{* * *} P<0.001$.

cause uncontrolled breakdown of the newly formed ECM and impair the healing process. It is therefore crucial to maintain the activity of the collagenases during the tissue reconstruction but interfere with their pathophysiologically persistent activation. One of the strategies for chronic wound treatment is based on the removal of these enzymes from the wound exudates by enzyme adsorption onto the dressing material [23]. Therefore, in this study the extent of collagenase binding onto the developed collagen-based sponges was evaluated in vitro.

Although in many cases collagenase adsorption onto collagenous substrates occurs within $1 \mathrm{~h}$, the process is much slower on dense (e.g. cross-linked) matrices [24]. Therefore, to assure equilibrium binding the biopolymer sponges were incubated for $24 \mathrm{~h}$ with the enzyme in concentration not causing degradation of the materials (results not shown), and the activity of the supernatants were further determined. All enzymatic solutions incubated with the sponges displayed lower activities compared to the control, meaning that collagenase was adsorbed on all specimens (Fig. 3A). As expected, the extent of binding decreased with the decrease of the substrate (collagen) content in the sponges. The protein adsorption on the cross-linked collagen was 52\%, while the addition of HA to the blends led to $38 \%$ protein adsorption on CHA6, 22\% on both CHA830 and CHA2000, and only 9\% on CHACS sponges.

Despite that the extent of collagenase adsorption differed between the experimental groups, an in vitro test for material biostability revealed similar (>95\%) resistance to collagenase $\left(10 \mu \mathrm{g} \mathrm{ml}^{-1}\right)$ degradation for all cross-linked biopolymer sponges (Fig. 3B).
However, using higher enzyme concentration $\left(60 \mu \mathrm{g} \mathrm{ml}^{-1}\right)$ made it possible to distinguish between the enzymatic sensitivity of different experimental groups, the results being in agreement with the enzymatic adsorption tests. More than $30 \%$ of the protein was digested from the pure collagen sponge, whereas the biostability of the sponges significantly increased after addition of HA and CS $(P<0.01$ for CHA6, $P<0.001$ for CHA830, CHA2000 and CHACS). Also, it is worth mentioning that even when higher enzyme concentration was used, all sponges could preserve their structural integrity during incubation-an important property of a dressing material that allows for its easy removal from the wound site.

It is known that the susceptibility of insoluble substrates, e.g. biopolymer matrices, to enzymatic transformations is inversely proportional to the cross-linking degree of the material. Higher cross-linking density of the substrate results in reduced surface area [14,25], lower accessibility of the enzyme to the binding sites and consecutively limited enzymatic adsorption and efficiency [26]. Thus, the inclusion of more cross-linking points (amino and carboxylic groups) by the addition of HA and CS to the collagenbased sponge resulted in improved biostability of the materials, but limited collagenase adsorption.

\subsection{Swelling of the cross-linked biopolymer sponges}

The ability of the dressing materials to absorb physiological fluids proved to be an important index for the assessment of their efficacy. The most important parameters influencing the swelling
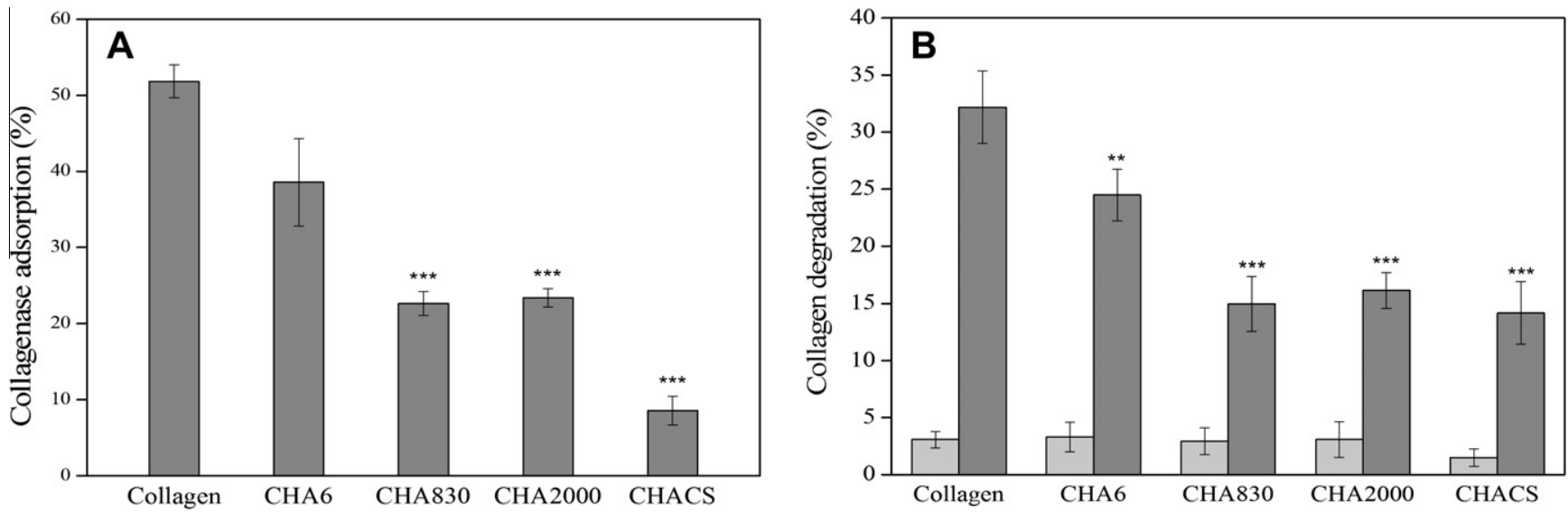

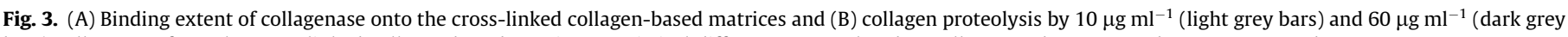
bars) collagenase from the cross-linked collagen-based matrices. Statistical differences are related to collagen and represented as ${ }^{* *} P<0.01$ and ${ }^{* * *} P<0.001$. 


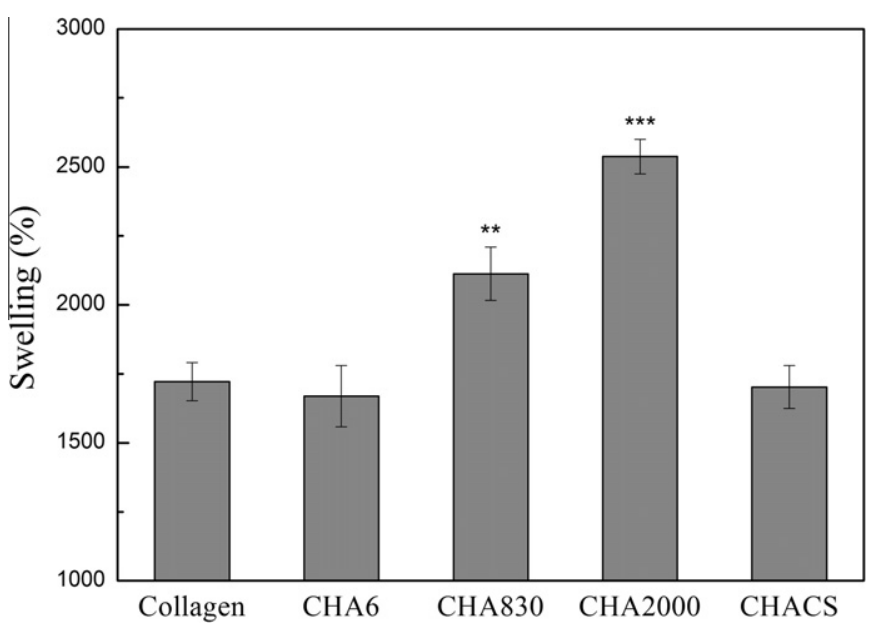

Fig. 4. Swelling of the cross-linked biopolymer matrices after $24 \mathrm{~h}$ incubation in PBS ( $\mathrm{pH}$ 7.4). Statistical differences are related to collagen and represented as ${ }^{* *} P<0.01$ and ${ }^{* * *} P<0.001$.

of the biopolymer constructs are the hydrophilicity of its constituents and the extent of cross-linking in the matrix [27]. However, owing to the diverse and dynamic nature of all chronic wounds, there is no general recommendation for the fluid uptake capacity of the corresponding dressings as long as the material maintains its form and stability after swelling to be easily removed from the wound site. In this study the addition of HA of different Mw to collagen altered differently the swelling of the CHA systems without causing a significant volume expansion of the specimens (Fig. 4). While CHA6 did not differ from the cross-linked collagen in swelling, the addition of HA with higher molecular weights resulted in a significant increase in fluid uptake $(P<0.01$ and $P<0.001$ for CHA830 and CHA2000, respectively). The presence of HA in collagen-based sponges is expected from one side to increase the swelling due to the high hydrophilicity of this polymer, but from another, to increase the cross-linking density of the system and affect negatively the swelling [19]. Although the results from the biostability test and collagenase binding studies indicated higher cross-linking densities in the specimens prepared with HA of higher $\mathrm{Mw}$, the latter retained more fluid compared to CHA6. The high affinity of HA for physiological fluids originates from the high density of negative charges that bind a cloud of cations, most notably $\mathrm{Na}^{+}$(here from PBS), which are osmotically active and promote swelling [28]. In order to estimate the anionic character of HA used in the study, the $\zeta$-potentials of HA solutions were determined (Nano-ZS, Malvern, UK). These values for HAs of 6 , 830 and $2000 \mathrm{kDa}$ were $-5.3 \pm 1.2,-17.1 \pm 2.2$ and $-16.6 \pm 1.1$ respectively. Thus, it can be hypothesized that HA with higher Mw would retain more fluid compared to HA of low Mw and, in spite of its densely cross-linked structure, would enhance the hydrophilic properties of the CHA system. The addition of CS to CHA830 decreased the swelling capacity of the specimen due to further cross-linking reactions with the amino groups from CS [29].

\subsection{In vitro evaluation of cell viability and morphology}

Although fibroblasts were metabolically active on all biopolymer matrices over the studied period ( $72 \mathrm{~h}$ ), some differences were observed among the experimental groups (Fig. 5). The results clearly demonstrated the effect of the HA molecular weight on the cells' viability: while there was no significant difference in viability after adding low molecular weight $\mathrm{HA}(6 \mathrm{kDa})$, the values were significantly $(P<0.001)$ lower for the sponges containing longer HA chains (CHA830, CHA2000 and CHACS). Moreover, the

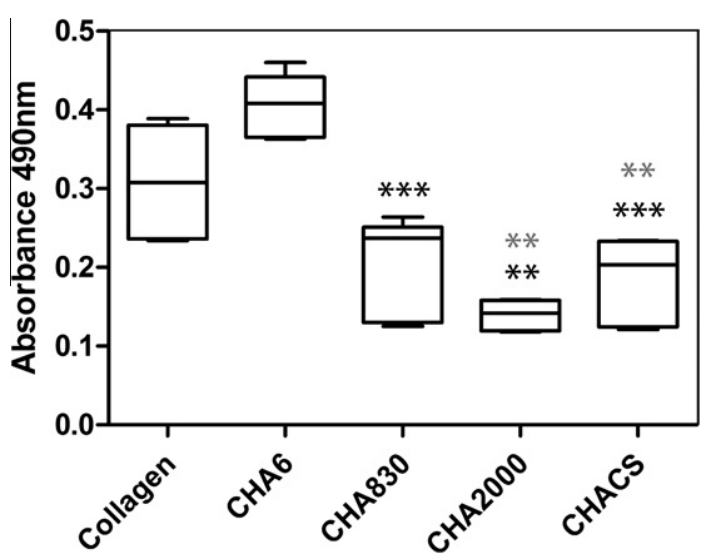

Fig. 5. Fibroblast viability after 3 days of culture in contact with the biopolymer sponges. Statistical differences are represented as ${ }^{*} P<0.05,{ }^{* *} P<0.01$ and ${ }^{* * *} P<0.001$, in grey or black, if related to collagen or CHA6, respectively.

cells cultured in contact with the collagen, CHA6 and CHACS sponges showed progressive increase in their metabolic activity during the whole period of culture (Sup. Fig. 1). However, the addition of HA with higher molecular weight (CHA830 and CHA2000) resulted in an increase in the metabolic activity only during the first $48 \mathrm{~h}$, which then was maintained or decreased. The reduced cell viability in the specimens containing longer HA chains can be assigned to their increased hydrophilicity related with reduced fibroblast cell growth [30]. On the other hand, this study confirms that different molecular weights of HA can be used to tune cell differentiation [31] and rule different cell responses [32].

The SEM analysis supported the results obtained by MTS assay: after $24 \mathrm{~h}$ of culture, adherent cells could be observed on all crosslinked sponges, being able to migrate inside the specimens and colonize the interiors (Sup. Fig. 2). At the end of the culture period, the amount of cells visible in the SEM images further increased (Fig. 6), confirming the relation between cell proliferation and their metabolic activity. It is also worth noting the different morphology of the cells cultured for $72 \mathrm{~h}$ on different specimens: when cultured on collagen sponge, fibroblasts show typical spindle morphology, establishing several contacts with neighbouring cells. More elongated cells are visible on CHA6 and CHA830, while round cells, indicating a poor attachment to the surface of the sponge, are observed for CHA2000 and CHACS. The amount of cells detected in CHACS sponges is remarkably lower when compared to the other specimens, due to the presence of chitosan in the formulation, related with the poor cell adhesion in our previous studies [33,34].

Finally, although no histological assay was carried out, the matrices with different composition are expected to provoke different tissue reactions. Whereas chitosan will most likely induce multinucleated giant cell formation [35], collagen will induce a mononuclear cellular tissue response including anti-inflammatory macrophages and low vascularization rates [36]. However, the latter does not mean that the collagen-based materials will not integrate well with the targeted tissue, but rather that the rapid transmembrane vascularization will not occur. With such response the clinical complications associated with the retrieval of the material after guided tissue regeneration is avoided.

\subsection{In vitro assessments of the sponge-like dressings loaded with polyphenols}

The multifactorial nature of all chronic wounds, characterized by prolonged inflammation and deleterious concentrations of oxidative and proteolytic enzymes, emphasizes the necessity for 

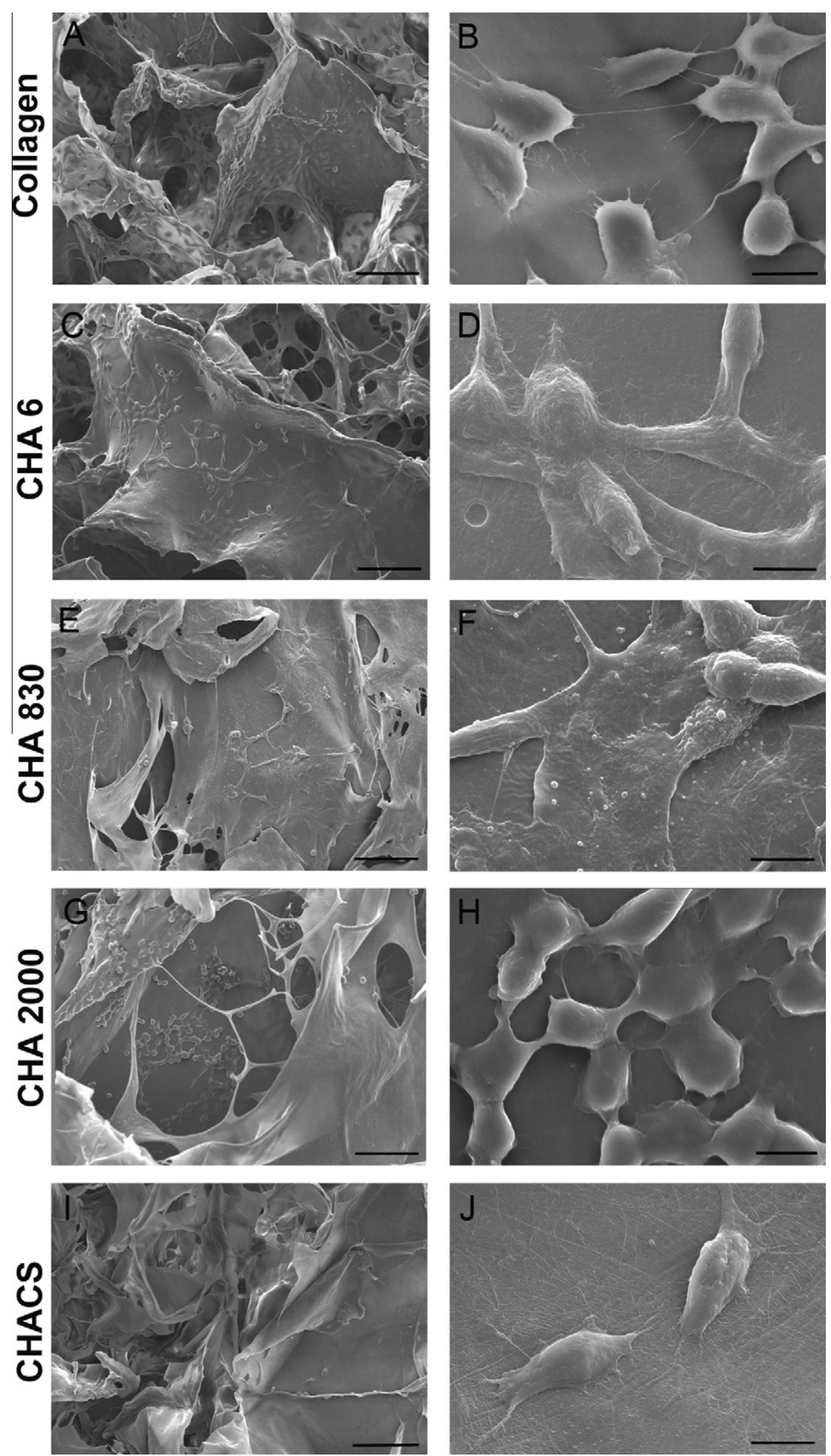

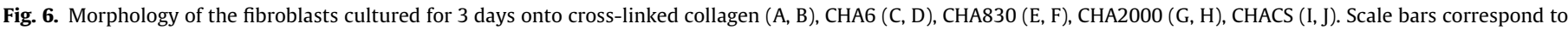
$100 \mu \mathrm{m}$ (left-side images) and $10 \mu \mathrm{m}$ (right-side images).

investigation of dressing materials with the ability to actively modulate the wound environment at molecular level and stimulate the healing. To this end, the cross-linked biopolymer sponges were impregnated with bioactive molecules, e.g. plant polyphenols, and evaluated in vitro as chronic wound dressings. Due to the large number of experimental groups, for simplicity only the results for polyphenol-loaded collagen, CHA830 and CHACS specimens are given.
3.5.1. Cytotoxicity evaluation (MTS) of the polyphenols-loaded sponges As antiproliferative effect of tea polyphenols for both cancer and normal cells has been previously reported [37], in this study the cytotoxicity of the obtained matrices before and after loading them with the polyphenols were compared. Generally, the loading of the sponges with polyphenols in the concentration used (1 $\mathrm{mM})$ did not induce a significant difference in the cytotoxicity of the matrices; the only significantly lower metabolic activities 


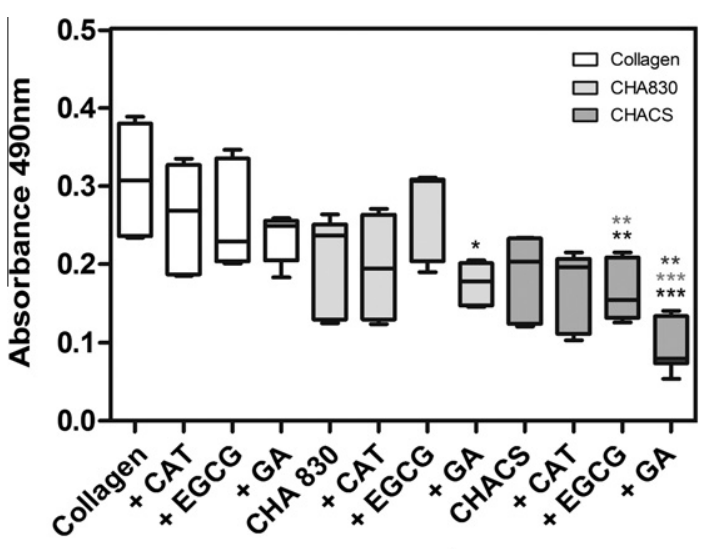

Fig. 7. Effect of polyphenols on fibroblast viability after 3 days in culture. Statistical differences are represented as ${ }^{*} P<0.05$, ${ }^{* *} P<0.01$ and ${ }^{* * *} P<0.001$, in black, light grey or dark grey, if related to collagen, CHA830 or CHACS, respectively.

$(P<0.01)$ were found for the cells cultured on the GA-loaded composite sponges (Fig. 7). Thus, the overall results for the biocompatibility demonstrated that the composition of the biopolymer-based material can have a stronger influence on cell viability than functional polyphenolic compounds per se.

\subsubsection{In vitro stability-at-use and swelling of the dressings}

The treatment of cross-linked biopolymer sponges with plant polyphenols had a different effect on the biostability of the sponges against collagenase $\left(60 \mu \mathrm{g} \mathrm{ml}^{-1}\right)$ degradation depending on the compound used (Fig. 8A). Specimens loaded with EGCG displayed the lowest degradation (less than 5\%) regardless of their biopolymer composition. Although not as efficient as EGCG, CAT also brought about a significant increase in the dressing resistances to enzymatic degradation. In this case, the values for collagen degradation obtained for experimental groups with different biopolymer composition followed the same trend as in the case of dressings without polyphenols. Treatment with GA did not affect the material's biostability, which is in good agreement with literature [38]. This result in combination with cytotoxicity inducing effects, though rather low, makes GA the least appropriate polyphenolic compound used in this study for functionalization of the biopolymer sponges.
Table 1

$I C_{50}$ values for MPO and collagenase inhibition by polyphenols.

\begin{tabular}{lcl}
\hline Polyphenol compound & $I C_{50}$ collagenase $(\mu \mathrm{M})$ & $I C_{50} \mathrm{MPO}(\mu \mathrm{M})$ \\
\hline EGCG & $92 \pm 8$ & $15 \pm 1$ \\
CAT & $152 \pm 11$ & $21 \pm 2$ \\
GA & $298 \pm 23$ & $29 \pm 1$ \\
\hline
\end{tabular}

Some phenolic compounds exert a well-known improvement in the stability of protein/polysaccharide matrices towards enzymatic degradation through phenols-biopolymers interactions [12,39] and enzyme inhibition [11]. The extent of biostability improvement is proportional to the number of phenol moieties in the polyphenolic compound [15]. Thus, the highest potential of EGCG to stabilize biopolymer sponges could be related to its high number of phenolic groups able to react with the biopolymers (Fig. 8B), and its highest potential for collagenase inhibition (Table 1). Although GA binds stronger to the sponges compared to CAT, the higher potential of CAT to inhibit collagenase still provided improved biostability of the materials. In conclusion, EGCG and CAT were found to effectively inhibit the proteolytic degradation of collagen, and thus have potential to partially compensate the loss of material biostability.

On the other hand, loading of the biopolymer matrices with polyphenols did not cause changes in the swelling behaviour of any experimental group (results not shown). Their concentration used in this study ( $1 \mathrm{mM}$ ) was probably too low to influence the swelling capacity of the cross-linked sponges.

\subsubsection{Myeloperoxidase inhibition and durability of the inhibition effect}

As a part of the immune response, MPO plays a major role in many diseases by catalysing the formation of the potent antimicrobial oxidant $\mathrm{HOCl}$ from $\mathrm{Cl}^{-}$in the presence of $\mathrm{H}_{2} \mathrm{O}_{2}$. However, the persistent activity of the $\mathrm{MPO}-\mathrm{H}_{2} \mathrm{O}_{2}$ system during prolonged inflammation, such as in chronic wounds, and the consequent $\mathrm{HOCl}$ accumulation at the wound site, may adversely affect the tissue regeneration [40]. It is therefore essential that the chronic wound treatment includes controlled MPO inhibition, which may also contribute to the control of the proteolytic activity [41]. Plant polyphenols exert their beneficial effect acting as both MPO inhibitors and MPO substrates, thereby deviating the enzyme from its catalytic cycle of $\mathrm{HOCl}$ generation [13].

Since the inhibition of the MPO activity by biopolymer platforms loaded with an active agent is related to its release profile

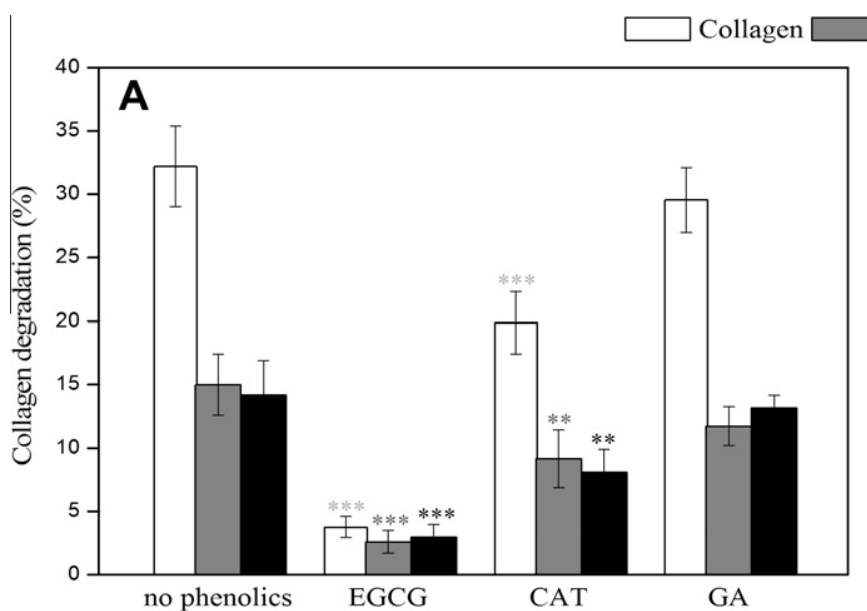

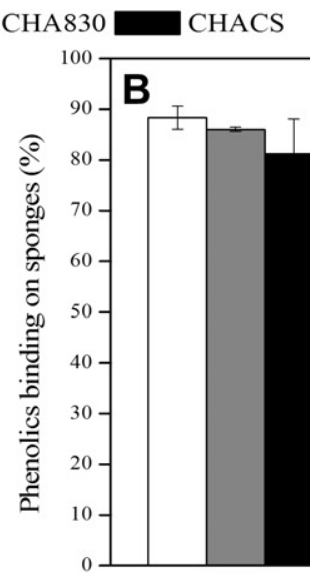

EGCG

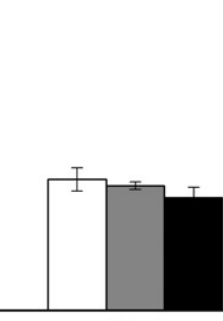

CAT

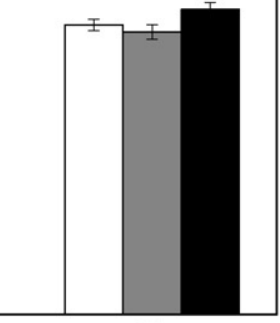

GA

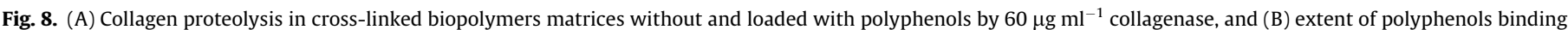

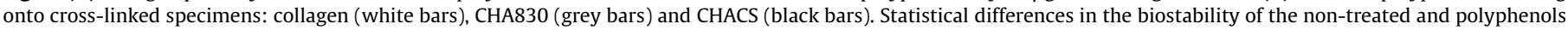
loaded specimens are represented as ${ }^{* *} P<0.01$ and ${ }^{* * *} P<0.001$, in light grey, dark grey and black if related to collagen, CHA830 and CHACS, respectively. 

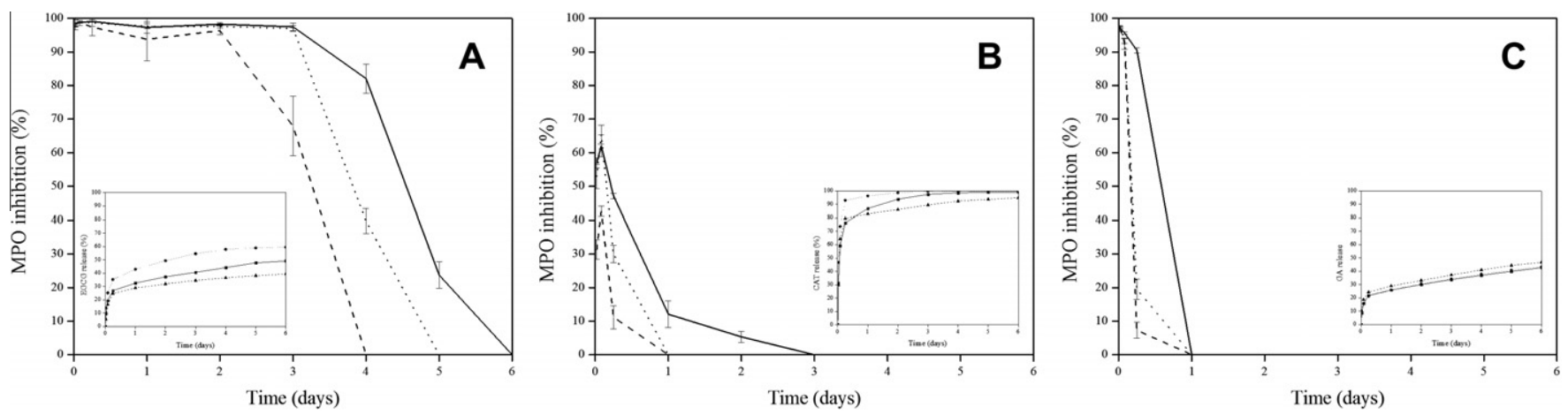

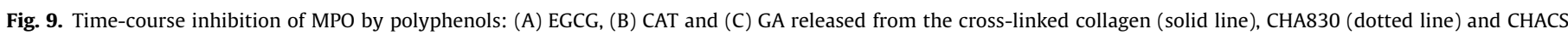
(dashed line). The inset graphs show the polyphenol release patterns from the corresponding biopolymer composite matrices.

[14], the MPO inhibition caused by the released polyphenols was investigated in vitro. In order to simulate the chronic inflammation, i.e. continuous influx of neutrophil derived MPO to the wound site, at each reading point the incubation solutions were replaced with new solutions containing the fresh enzyme. Thus, the MPO inhibition in each reading point corresponds only to the effect of the polyphenols released between the two reading points. The durability of such effect was evaluated up to 6 days.

Although CAT reduces stronger than GA the MPO activity (Table 1 ), the lowest extent of CAT binding on the materials (Fig. 8B) might have led to the least efficient initial MPO inhibition with CAT-loaded dressings (Fig. 9). Moreover, except for CAT-loaded collagen, all GA- and CAT-loaded dressings inhibited MPO only up to $6 \mathrm{~h}$ despite that their release continued after this point (Fig. $9 \mathrm{~B}$ and C). The possible reasons for such short period of efficiency could be: (i) a large part of the active agent was released within the first few hours as in the case of CAT-loaded sponges (Fig. 9B inset), and (ii) the initial burst release was followed by a slow release of phenolics, apparently insufficient to achieve an inhibitory effect as in the case of GA-loaded sponges (Fig. 9C inset). On the other hand, the pattern for EGCG was of initial burst release followed by a sufficient for MPO inhibition sustained release (Fig. 9A inset). The highest inhibitory potential of EGCG-loaded systems was also supported by the high binding affinity of EGCG onto the biopolymer sponges (Fig. 8B). The EGCG released from CHACS sponge maintained the MPO inhibitory capacity up to 3 days, whereas when released from CHA830 and collagen alone the effect was prolonged up to 4 and 5 days, respectively (Fig. 9A). Strong complexation of polyphenols with chitosan at neutral $\mathrm{pH}$ was possibly the reason for the slowest release pattern from CHACS sponge [39].

\section{Conclusions}

An efficient chronic wound treatment approach requires the application of stable-at-use hydrophilic dressing platforms with good mechanical performance augmented with active functions to control the inflammation-related enzymatic activities at the wound site. In this study freeze-dried biopolymer composite sponges were produced from collagen, collagen/HA and collagen/ $\mathrm{HA} / \mathrm{CS}$ and further stabilized by cross-linking with EDAC through amide- and ester-bond formation, whereas the functionalization was carried out by impregnating them with natural polyphenols. The physico-mechanical properties of the biocomposite matrices were easily adjustable by altering their composition, i.e. through the formation of additional cross-links after collagen blending with $\mathrm{HA}$ and CS. The composite sponges also exhibited improved biostability compared to the collagen one. Whereas all specimens with or without polyphenols were able to retain more than 17-fold of their own weight of physiological fluid without deformation, improved swelling was achieved only by the addition of HA with higher Mw. The in vitro assessments of fibroblast behaviour indicated that although cells are able to adhere to all sponges, they were adversely affected by the presence of HA macromolecules and CS. Moreover, the addition of polyphenols to blended sponges affects cells to a lesser extent than the composition of the material itself. Finally, the specimens impregnated with polyphenols efficiently inhibited the collagenase and MPO activities in vitro. The duration of the MPO inhibition effect could be tuned by the biopolymer composition of the materials and proper selection of the polyphenolic compound. The possibility to control the activities of major wound enzymes during prolonged periods makes these active composite dressings potentially useful in chronic wound treatment. Given the aggregate results for the material's bulk properties, cytotoxicity and capacity for the enzyme inhibition, the EGCG-loaded CHA composition containing higher Mw hyaluronic acid showed the highest potential for the targeted application.

\section{Acknowledgements}

This work was carried out under the scope of the EU projects Lidwine (Contract No. FP6-026741) and Find\&Bind (Contract No. FP7-229292). The PhD grant BES-2008-00374 from the Spanish Ministerio de Ciencia e Innovación (MICINN) is gratefully acknowledged.

\section{Appendix A. Supplementary data}

Supplementary data associated with this article can be found, in the online version, at http://dx.doi.org/10.1016/j.actbio.2012.10. 014 .

\section{References}

[1] Trengove NJ, Stacey MC, Macauley S, Bennett N, Gibson J, Burslem F, et al. Analysis of the acute and chronic wound environments: the role of proteases and their inhibitors. Wound Repair Regen 1999;7:442-52.

[2] Rojkind M, Domínguez-Rosales JA, Nieto N, Greenwel P. Role of hydrogen peroxide and oxidative stress in healing responses. Cell Mol Life Sci 2002;59:1872-91.

[3] Ovington L. Advances in wound dressings. Clin Dermatol 2007;25:33-8.

[4] Cullen B, Smith R, McCulloch E, Silcock D, Morrison L. Mechanism of action of PROMOGRAN, a protease modulating matrix, for the treatment of diabetic foot ulcers. Wound Repair Regen 2002;10:16-25.

[5] Jarman-Smith M, Bodamyali T, Stevens C. Howell J, Horrocks M, Chaudhuri J. Porcine collagen crosslinking, degradation and its capability for fibroblast adhesion and proliferation. J Mater Sci: Mater Med 2004;15:925-32.

[6] Hsieh Y-S, Yang S-F, Lue K-H, Chu S-C, Lu K-H. Effects of different molecular weight hyaluronan products on the expression of urokinase plasminogen activator and inhibitor and gelatinases during the early stage of osteoarthritis. J Orthop Res 2008;26:475-84 
[7] Ueno H, Yamada H, Tanaka I, Kaba N, Matsuura M, Okumura M, et al. Accelerating effects of chitosan for healing at early phase of experimental open wound in dogs. Biomaterials 1999;20:1407-14.

[8] Harley BA, Leung JH, Silva ECCM, Gibson LJ. Mechanical characterization of collagen-glycosaminoglycan scaffolds. Acta Biomater 2007;3:463-74.

[9] Lin Y-C, Marra KG, Jan S-S, Liu D-C. Synthesis and characterization of collagen/ hyaluronan/chitosan composite sponges for potential biomedical applications. Acta Biomater 2009;5:2591-600.

[10] Everaerts F, Torrianni M, Hendriks M, Feijen J. Biomechanical properties of carbodiimide crosslinked collagen: influence of the formation of ester crosslinks. J Biomed Mater Res A 2008;85A:547-55.

[11] Haslam E. Natural polyphenols (vegetable tannins) as drugs: possible modes of action. J Nat Prod 1996;59:205-15.

[12] Madhan B, Subramanian V, Rao JR, Nair BU, Ramasami T. Stabilization of collagen using plant polyphenol: role of catechin. Int J Biol Macromol 2005;37:47-53.

[13] Díaz-GonzáLez M, Rocasalbas G, Francesko A, Touriño S, Torres JL, Tzanov T. Inhibition of deleterious chronic wound enzymes with plant polyphenols. Biocatal Biotransform 2012;30:102-10.

[14] Francesko A, Rocasalbas G, Touriño S, Mattu C, Gentile P, Chiono V, et al. Crosslinked collagen sponges loaded with plant polyphenols with inhibitory activity towards chronic wound enzymes. Biotechnol J 2011;6:1208-18.

[15] Madhan B, Krishnamoorthy G, Rao JR, Nair BU. Role of green tea polyphenols in the inhibition of collagenolytic activity by collagenase. Int J Biol Macromol 2007;41:16-22.

[16] Kesava Reddy G, Enwemeka CS. A simplified method for the analysis of hydroxyproline in biological tissues. Clin Biochem 1996;29:225-9.

[17] Edwards CA, O'Brien Jr WD. Modified assay for determination of hydroxyproline in a tissue hydrolyzate. Clin Chim Acta 1980;104:161-7.

[18] Singleton VL, Orthofer R, Lamuela-Raventós RM, Lester P. [14] Analysis of total phenols and other oxidation substrates and antioxidants by means of folinciocalteu reagent. Methods Enzymol 1999:152-78 [Academic Press].

[19] Davidenko N, Campbell JJ, Thian ES, Watson CJ, Cameron RE. Collagenhyaluronic acid scaffolds for adipose tissue engineering. Acta Biomater 2010;6:3957-68.

[20] Van Wart HE, Steinbrink DR. A continuous spectrophotometric assay for Clostridium histolyticum collagenase. Anal Biochem 1981;113:356-65.

[21] Park S-N, Park J-C, Kim HO, Song MJ, Suh H. Characterization of porous collagen/ hyaluronic acid scaffold modified by 1-ethyl-3-(3-dimethylaminopropyl) carbodiimide cross-linking. Biomaterials 2002;23:1205-12.

[22] Madhavan K, Belchenko D, Motta A, Tan W. Evaluation of composition and crosslinking effects on collagen-based composite constructs. Acta Biomater 2010;6:1413-22.

[23] Edwards JV, Howley PS. Human neutrophil elastase and collagenase sequestration with phosphorylated cotton wound dressings. J Biomed Mater Res A 2007;83A:446-54.

[24] Metzmacher I, Radu F, Bause M, Knabner P, Friess W. A model describing the effect of enzymatic degradation on drug release from collagen minirods. Eur J Pharm Biopharm 2007;67:349-60.

[25] Yan L-P, Wang Y-J, Ren L, Wu G, Caridade SG, Fan J-B, et al. Genipin-crosslinked collagen/chitosan biomimetic scaffolds for articular cartilage tissue engineering applications. J Biomed Mater Res A 2010;95A:465-75.
[26] Metzmacher I, Ruth P, Abel M, Friess W. In vitro binding of matrix metalloproteinase-2 (MMP-2), MMP-9, and bacterial collagenase on collagenous wound dressings. Wound Repair Regen 2007;15:549-55.

[27] Adekogbe I, Ghanem A. Fabrication and characterization of DTBP-crosslinked chitosan scaffolds for skin tissue engineering. Biomaterials 2005;26: 7241-50.

[28] Dhanasingh A, Salber J, Moeller M, Groll J. Tailored hyaluronic acid hydrogels through hydrophilic prepolymer cross-linkers. Soft Matter 2010;6:618-29.

[29] Ma L, Gao C, Mao Z, Zhou J, Shen J, Hu X, et al. Collagen/chitosan porous scaffolds with improved biostability for skin tissue engineering. Biomaterials 2003;24:4833-41.

[30] Wang YW, Wu Q Chen GQ Reduced mouse fibroblast cell growth by increased hydrophilicity of microbial polyhydroxyalkanoates via hyaluronan coating. Biomaterials 2003;24:4621-9.

[31] Maharjan AS, Pilling D, Gomer RH. High and low molecular weight hyaluronic acid differentially regulate human fibrocyte differentiation. PLoS One 2011;6:e26078

[32] Deed R, Rooney P, Kumar P, Norton JD, Smith J, Freemont AJ, et al. Earlyresponse gene signalling is induced by angiogenic oligosaccharides of hyaluronan in endothelial cells. Inhibition by non-angiogenic, highmolecular-weight hyaluronan. Int J Cancer 1997;71:251-6.

[33] Francesko A, Costa D, Lisboa P, Reis RL, Pashkuleva I, Tzanov T. GAGs-thiolated chitosan assemblies for chronic wounds treatment: control of enzyme activity and cell attachment. J Mater Chem 2012. http://dx.doi.org/10.1039/ C2JM31051.

[34] Lopez-Perez PM, Marques AP, Silva RMPD, Pashkuleva I, Reis RL. Effect of chitosan membrane surface modification via plasma induced polymerization on the adhesion of osteoblast-like cells. J Mater Chem 2007;17:4064-71.

[35] Muzzarelli RAA, Morganti P, Morganti G, Palombo P, Palombo M, Biagini G, et al. Chitin nanofibrils/chitosan glycolate composites as wound medicaments. Carbohydr Polym 2007;70:274-84.

[36] Ghanaati S. Non-cross-linked porcine-based collagen I-III membranes do not require high vascularization rates for their integration within the implantation bed: a paradigm shift. Acta Biomater 2012;8:3061-72.

[37] Weisburg JH, Weissman DB, Sedaghat T, Babich H. In vitro cytotoxicity of epigallocatechin gallate and tea extracts to cancerous and normal cells from the human oral cavity. Basic Clin Pharmacol Toxicol 2004;95:191-200.

[38] Jackson J, Zhao J, Wong W, Burt H. The inhibition of collagenase induced degradation of collagen by the galloyl-containing polyphenols tannic acid, epigallocatechin gallate and epicatechin gallate. J Mater Sci: Mater Med 2010;21:1435-43.

[39] Popa M-I, Aelenei N, Popa VI, Andrei D. Study of the interactions between polyphenolic compounds and chitosan. React Funct Polym 2000;45:35-43.

[40] Nicholls SJ, Hazen SL. Myeloperoxidase and cardiovascular disease. Arterioscler Thromb Vasc Biol 2005;25:1102-11.

[41] Fu X, Kassim SY, Parks WC, Heinecke JW. Hypochlorous acid oxygenates the cysteine switch domain of pro-matrilysin (MMP-7). J Biol Chem 2001;276:41279-87. 